OPEN ACCESS

Theory of Radiative Lifetime of an Activator lon due to Surface Plasmons

To cite this article: K. C. Mishra et al 2018 ECS J. Solid State Sci. Technol. 7 R42

View the article online for updates and enhancements. 


\title{
Theory of Radiative Lifetime of an Activator Ion due to Surface Plasmons
}

\author{
K. C. Mishra, ${ }^{1,2, *}$ J. Collins, ${ }^{3}$ and A. Piquette $\oplus^{4, * *, z}$ \\ ${ }^{1}$ OSRAM SYLVANIA Inc. (retired), Innovation, Beverly, Massachusetts 01915, USA \\ ${ }^{2}$ North Chelmsford, Massachusetts 01863, USA \\ ${ }^{3}$ Department of Physics and Astronomy, Wheaton College, Norton, Massachusetts 02766, USA \\ ${ }^{4}$ OSRAM SYLVANIA Inc., Innovation, Beverly, Massachusetts 01915, USA
}

\begin{abstract}
We consider the lifetime of a phosphor layer situated near a metallic film. We first make general observations of the effect of the plasmons on the lifetime and quantum efficiency of the luminescent ions in the phosphor layer and on the role of the plasmons in the emission process. The effect of the plasmons in the excitation process and on the total luminescence from the system is also discussed. We then derive an expression for the lifetime of the optical ions interacting with the surface plasmon modes using the Einstein $A$ and $B$ coefficient formalism, which requires a determination of the mode density of the surface plasmons. Because the surface plasmons represent a 2-dimensional system, a length parameter must be introduced into the expression of the mode density. We derive this parameter using a semi-classical approach, and show its dependence on the dielectric constants of the system and on the distance from the metallic layer.

(C) The Author(s) 2018. Published by ECS. This is an open access article distributed under the terms of the Creative Commons Attribution Non-Commercial No Derivatives 4.0 License (CC BY-NC-ND, http://creativecommons.org/licenses/by-nc-nd/4.0/), which permits non-commercial reuse, distribution, and reproduction in any medium, provided the original work is not changed in any way and is properly cited. For permission for commercial reuse, please email: oa@electrochem.org. [DOI: 10.1149/2.0081805jss]
\end{abstract}

(cc) BY-NC-ND

Manuscript submitted February 22, 2018; revised manuscript received April 18, 2018. Published May 4, 2018.

Recently there has been considerable interest on utilizing surface plasmons in metallic thin films to enhance internal quantum efficiency of luminescent materials ${ }^{1-12}$ and light emitting diodes (LED). ${ }^{13-22}$ The most important effect of the surface plasmons on luminescent ions is on their lifetime in the excited state which could contribute to improving the internal quantum efficiency of luminescent materials in the form of a slab or a layer. In this paper, we have studied how the change in the excited state lifetime of the radiating atoms could be described using the dispersion relations of the surface plasmons.

The lifetime of an excited ion, $\tau$ as measured by the decay measurements consists of two components, radiative lifetime, $\tau_{R}$ and nonradiative lifetime, $\tau_{N R}$ the reciprocals of which are additive,

$$
\frac{1}{\tau}=\frac{1}{\tau_{R}}+\frac{1}{\tau_{N R}}
$$

The radiative lifetime of an ion depends on the transition moments of the ion, i.e., the matrix element of the electric dipole and quadrupole moments or magnetic moments between the excited and ground state wavefunctions of the radiating ions. Normally the radiative lifetime is a characteristic of the optical ion and does not change unless the wavefunctions of the optical ions are perturbed. On the other hand, nonradiative lifetime is associated with the relaxation of the excited state to phonons, lattice defects and impurities with a loss of the excited state energy. It is sometimes possible to increase the nonradiative lifetime by controlling the purity of the material, operating temperature, better material processing etc., thus decreasing the probability of nonradiative relaxation of the ion. From Eq. 1, it can be shown that when the nonradiative lifetime increases, the lifetime of the excited state increases. This increase in lifetime usually results in an increase of the internal quantum efficiency.

If a new nonradiative pathway is opened, the nonradiative lifetime will decrease. It will not always decrease the internal quantum efficiency if the transferred energy is not completely lost as it is in the case of phonons. Such could be the case for surface plasmons that may be excited by both the radiation exciting the optical ion or emitted by the same.

Unlike the bulk plasmons, the surface plasmons in a thin metallic film could be excited by the electromagnetic waves if the surface of the metallic film is modified to compensate for any momentum

\footnotetext{
*Electrochemical Society Fellow.

**Electrochemical Society Member.

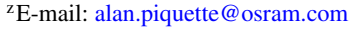

mismatch between the incident photons and surface plasmons. ${ }^{23-25}$ The subsequent change in the excited state lifetime of the optical ion is caused by the attenuating electromagnetic fields associated with the surface plasmons. In other words, an excited optical ion could relax to the ground state through the surface plasmon modes, in addition to the vacuum modes. This relaxation process will be referred to as a nonradiative transition, since there is no energy radiated into the far field. We note, however, that unlike nonradiative decay into phonons, the energy transferred to the surface plasmons is stored as electromagnetic energy and may lead to radiation. This description of the interaction between a radiating ion and a metallic film through the surface plasmon modes allows one to treat the nonradiative energy transfer as a spontaneous decay process using standard quantum mechanical methods.

Before we develop the theory of interaction between the radiating atoms and the surface plasmons, let us first consider what happens to the energy transferred nonradiatively to the surface plasmon modes. Unlike in the case of energy loss to phonons, a part or all of the transferred energy could be back-transferred to the excited ions, or re-radiated. In certain cases, there could be an increase of the internal quantum efficiency of the luminescent material even if the lifetime of the optical ions is shortened. It is not always possible to predict if a metallic film in the vicinity of a layer of luminescent materials would enhance its quantum efficiency in an a priori manner. It is for this reason we have simulated quantum efficiencies of a luminescent layer in the vicinity of a thin metallic film (Figure 1) considering scenarios of re-radiation and back-transfer of the transferred energy.

Let $N$ and $N_{p}$ represent the number of activators ions in the excited state, and the number of surface plasmons excited through relaxation of the activator ions via the plasmon modes. $P_{r a d}, P_{p h}, P_{p l}$ and $P_{b t}$ represent transition rates through emission of radiation, phonons, plasmons and back-transfer rate from plasmons to ions respectively. Let us assume further that $\eta$ represents the quantum efficiency of the system, phosphor plus metallic layer.

Let us assume that a fraction, $\alpha$ of the excited plasmons re-radiate after being excited. Under steady state conditions, the quantum efficiency of the system, $\eta$ can be shown to be

$$
\eta=\left(\frac{1}{P_{r a d}+P_{p h}+P_{p l}}\right)\left(P_{r a d}+\alpha P_{p l}\right)
$$

Let $\eta_{0}$ represent the quantum efficiency of the phosphor in the absence of the metallic layer, i.e., $P_{p l}=0$. Using Eq. 2, the following cases could be demonstrated: 


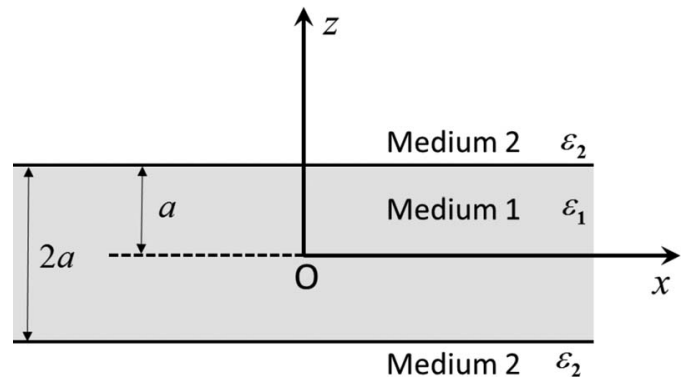

Figure 1. Schematic representation of semi-infinite metallic slab (Medium 1) with dielectric constant $\varepsilon_{1}$ surrounded by a dielectric material (Medium 2) with dielectric constant $\varepsilon_{2}$

Case I. If $P_{p h}$ is zero, $\eta_{0}=1.0$ and $\eta$ will always be less than or equal to $\eta_{0}$, i.e., $\eta \leq \eta_{0}$. A part of the energy transferred to the plasmons will be lost nonradiatively through the intrinsic loss mechanisms such as the Joule heating. For a highly efficient phosphor, such as $\mathrm{Y}_{2} \mathrm{O}_{3}: \mathrm{Eu}^{3+}$, any plasmonic interaction will lead to a lower quantum efficiency and the lifetime will always decrease due to relaxation of the excited state through the plasmonic modes.

Case II. If $P_{p h}=P_{p l}=P_{r a d}, \eta_{o}=0.5$ but $\eta$ will change linearly for $\alpha \in[0,1]$ from 0.33 to 0.67 . This case shows the general result that for phosphors with low quantum efficiency, there is a possibility that $\eta$ will increase with decreasing lifetime due to the plasmonic interactions.

Next, we consider how the quantum efficiency behaves under the following conditions.

(1) The plasmons may transfer energy back to the excited ions, and

(2) there is no direct emission from the plasmon and no losses in the metallic layer.

In this scenario, one needs to solve the following rate equations in order to calculate $\eta$ :

$$
\begin{gathered}
\frac{d N}{d t}=-N \mathrm{P}_{r a d}-N P_{p l}+N_{p} P_{b t} \\
\frac{d N_{p}}{d t}=N P_{p l}-N_{P} P_{b t}
\end{gathered}
$$

Using Eqs. 3-4, we have considered the following cases: Case III, where there are no phonon losses and Case IV, where phonon losses are present. They are discussed below:

Case III. This is the case where $\eta_{0}=1.0$ : the ion decays purely radiatively in the absence of a metallic surface. This is a trivial case, because in Equations 3 and 4 the only intrinsic losses are due to the ion decaying nonraditively into phonons. If we exclude those losses, then the quantum efficiency will always be unity, independent of the relative rates of the other mechanisms.

Case IV. For situations where the rate of phonon decay is non-zero, the following three scenarios present the basic findings.

$$
\begin{gathered}
P_{\text {rad }}=P_{p h}=P_{p l}=P_{b t}=10^{3}, \eta=\eta_{o}=0.5 \\
P_{\text {rad }}=\frac{P_{p h}}{10}=\frac{P_{p l}}{10}=\frac{P_{b t}}{10}=10^{3}, \eta=\eta_{o}=0.091 \\
P_{\text {rad }}=P_{p h}=\frac{P_{p l}}{10}=\frac{P_{b t}}{10}=10^{3}, \eta=\eta_{o}=0.5
\end{gathered}
$$

In each of these scenarios, the phosphor has quantum efficiency less than unity. The value of the quantum efficiency is always the same as that of the ions in the absence of a metallic surface, which is a consequence of our assumption that the plasmons do not radiate. Different for each scenario is the average time required for the excited ion to radiate away its energy into the far field.
Cases III and IV show that if the plasmons do not radiate, it is not possible to increase the quantum efficiency of the system. Thus, any increase in the observed quantum efficiency of the system is not due to increase in radiation from the luminescent ion; rather it is due to emission from the surface plasmons. The presence of the metallic surface will generally decrease the lifetime of the ion. Whether or not that decrease in the lifetime is accompanied by an increase in the emission depends not on the ion, but on the ability of the plasmon field to radiate before plasmon losses (ohmic, scattering, etc.) rob the energy from the plasmon field. Any losses, whether they occur at the site of the ion or on the metallic surface, will decrease the quantum efficiency of the system.

Since measuring the lifetime is experimentally a much easier task than measuring the quantum efficiency, it is useful to consider the interpretation of the measured lifetime of a luminescent ion in the presence of a metallic surface. Equation 1 tells us that the lifetime of the ion necessarily decreases in the presence of a metallic surface, due to the additional non-radiative decay channel opened up by the presence of the surface plasmons. Also, it is important to note that the plasmons excited by the luminescent ion are at the same frequency (or frequencies) as the emission from the ion. In measuring the lifetime, it is usually impossible to distinguish photons emitted by the ions from those emitted by the plasmons. Thus, in a lifetime measurement, one is necessarily detecting photons emitting from two different systems, the ions and the plasmons. The observed decay pattern contains information on both systems. Strictly speaking, one is not measuring the lifetime of the ions or of the plasmons, but rather the lifetime of the combined system. In practice, however, the lifetime of the ion is typically in the ns to ms range, while the lifetime of a plasmon is typically on the order of ps, or even less. Thus, when making a lifetime measurement, the measured decay curve will follow closely the decay curve of the ions, even if most of the photons are being emitted from the metal. Caution must be taken, however, in interpreting the lifetime data, because any decrease in the lifetime of the ion is simply due to the additional decay channels opened up due to the presence of the surface plasmon field. But a decrease in the lifetime does necessarily imply an increase in the quantum efficiency. Losses due to phonons (and perhaps other losses) at the site of the ion and losses experienced by the surface plasmons, coupled as well as the radiative rates of emission of the ions and the plasmons all help determine whether the decrease in the observed lifetime will lead to an increase in the luminescence output.

The ion-plasmon interaction could also occur at the wavelength of the exciting radiation because of the electromagnetic nature of the plasma oscillations. This could lead to an enhancement in the emission intensity, particularly for a stack of dielectric-metal-phosphor layers, and could be definitively useful in many applications, although there will be no change in the lifetime of the optical ion. Due to the Stokes shift, an optical ion in a solid is always excited at an energy higher than that of the emitted photon. Even though the excitation and emission processes of an ion involve strictly two electronic energy levels, the excited electron traverses through many vibronic energy levels before emitting a photon. These vibronic levels are a consequence of the vibrational degrees of freedom in the lattice. The vibronic energy levels associated with the excitation and emission peaks together with that of the ground state could be thought of as a three level canonical atomic system. An ion first gets excited to a higher vibronic level, then relaxes to a lower energy, emitting vibronic level through phonon generation, and finally returns to the ground state emitting a photon. Thus, the number of photons emitted by an optical ion depends on both the intensity of the exciting radiation to reach the top vibronic energy level and the radiative lifetime of the emitting vibronic level.

Let us consider a stack of dielectric-metal-phosphor layer on which light is incident from the side of the phosphor layer. If the phosphor layer is not too thick, a part of the incident radiation could reach the metallic layer. This radiation could thus excite the plasmon modes corresponding to the frequency of the exciting radiation. These plasmons in turn would add to the electric field at the site of the optical ion and thus would increase the transition rate of the ion from the 
ground to the excited state. This effect is similar to increasing the intensity of incident radiation at the site of the ions. Thus, more ions will be promoted to the excited state compared to a situation with no metallic film being present. Therefore, even for an identical lifetime for the emitting state of the activator ion, there will be more photons emitted in response to the same incident radiation. The intensity of the emission in this reflectance mode of the configuration of dielectricmetal-phosphor layers would be observed to increase because of the plasmons exciting the ion in addition the photons. Clearly this mechanism for enhancing the light output from a layer of phosphor will depend on specific arrangements of the metallic and phosphor layers in the device and the mode of excitation.

The main focus of this manuscript is to explore how the radiative modes of an excited ion are enhanced by the surface plasmonic modes leading to a decrease in the excited state lifetime. We have investigated this process using a semiclassical approach describing the interaction of plasmon modes through the attenuating electromagnetic field of the surface plasmons with the radiating ions. Thus, the results obtained in the following sections are valid only when the separation between the metallic film and the radiating ions in the phosphor layer is such that the electromagnetic fields associated with the surface plasmons have not attenuated to zero at the site of the optical ions, and more importantly, the optical ions are at a distance from the metal such that the ions and the metallic film could be considered as two separate entities. The latter restriction on distance requires that this spatial separation between the optical ions and the metallic film should be an order of magnitude longer than the sum of van der Walls radii of atoms in the metallic layer and optical ions (10-100 ̊). At shorter distances, the physio-chemical reaction between the atoms in the phosphor layer and the metal film could affect the electronic states of the radiating ions and metal atoms. Therefore, the theory developed in the latter sections is applicable if the separation between the optical ions in the fluorescent layer and metal atoms are shorter than the wavelength of the emitting or exciting radiation and about an order of magnitude longer than the sum of their van der Walls radii. At longer distances, comparable to the wavelength of exciting or emitting radiation, the interference of the incoming and outgoing waves will be important and would lead to the Drexhage effect. ${ }^{26}$

In order to calculate the lifetime due to electron-plasmon interaction, one could think of the ion in the excited state as transferring energy into the plasmon modes, instead of the vacuum modes. Conceptually, this allows one to use the Einstein's $A$ - and $B$-coefficients to calculate the decay time due to interaction between the excited ion and the surface plasmons. Therefore, our approach to calculating the lifetime of optical ions is based on calculating directly the mode density of surface plasmons and using this mode density to express the Einstein $A$-coefficient for the radiating ions. The $A$-coefficient is reciprocal to the corresponding lifetime. In order to achieve this, first we expressed the $A$-coefficient in terms of the available mode densities including that of the surface plasmons. Since the mode density associated with the surface plasmons is that of a two dimensional system, a length parameter has to be introduced to use the formalism developed in this work. This length parameter for surface plasmon-optical ion interaction has been calculated using a semiclassical approach. The underlying approach could be easily extended to more complex configurations of phosphor-metallic layer composites and is intuitively appealing.

\section{Surface Plasmon Solutions for a Metal of Thickness, 2a}

First, we investigate the dispersion relation for the surface plasmons associated with a thin metallic film sandwiched between two similar dielectric layers, and the associated electromagnetic fields and their spatial attenuation behavior. The dispersion curves provide information regarding the mode density and the spatial attenuation behavior describes how thick the phosphor layer should be in order to experience these fields in addition to that of the incident radiation.
The electromagnetic fields associated with the surface plasmons of a metallic film have been extensively discussed in the literature. ${ }^{23}$ For the sake of consistency and clarity, we will provide a brief description of the divergence free solutions of the Maxwell equations for a semi-infinite metallic slab of thickness $2 a$ and dielectric constant $\varepsilon_{1}$ surrounded by a dielectric medium of dielectric constant $\varepsilon_{2}$ (Figure 1). The derivation here follows an approach from Madelung. ${ }^{27}$ The coordinate system for solving the Maxwell equations are chosen arbitrarily at a point $O$, half way between the two interfaces of the metallic slab (Figure 1). In this coordinate system, we seek solutions of the Maxwell equations in the Gaussian units:

$$
\begin{gathered}
\vec{\nabla} \cdot \vec{D}=0 \\
\vec{\nabla} \cdot \vec{B}=0 \\
\vec{\nabla} \times \vec{E}=-\frac{1}{c} \frac{\partial \vec{B}}{\partial t} \\
\vec{\nabla} \times \vec{H}=\frac{1}{c} \frac{\partial \vec{D}}{\partial t}
\end{gathered}
$$

Both media are considered charge free and nonmagnetic; therefore for both media,

$$
\mu=1 \Rightarrow \vec{B}=\vec{H}
$$

We consider a $p$-polarized solution propagating along the $x$-direction:

$$
\begin{gathered}
\vec{E}=\left(E_{x}(z) \hat{i}+E_{z}(z) \hat{k}\right) e^{i\left(k_{x} x-\omega t\right)} \\
\vec{H}=\left(H_{y}(z) \hat{j}\right) e^{i\left(k_{x} x-\omega t\right)}
\end{gathered}
$$

Here, $\hat{i}, \hat{j}$ and $\hat{k}$ represent unit vectors along $x$-, $y$ - and $z$-directions, and $k$ the wavevector of a propagating wave with frequency, $\omega(\omega$ is indeed the angular frequency as opposed to $v$ which is the frequency associated with the propagating waves. However, we will refer to these parameters as frequency interchangeably while the relationship between the two is given by $\omega=2 \pi \nu$ ). The $s$-polarized waves will not yield any surface polarization waves, and will not be considered further. The conditions of continuity of the electric and magnetic fields require that at the interfaces, $z=a$ and $z=-a$

$$
\begin{gathered}
E_{1 x}=E_{2 x} \\
\varepsilon_{1} E_{1 z}=\varepsilon_{2} E_{2 z} \\
H_{1 y}=H_{2 y}
\end{gathered}
$$

The subscripts, 1 and 2 will denote solutions corresponding to medium 1 and medium 2 respectively. Using Eqs. 5 and 10 , it can be readily shown that

$$
\frac{\partial E_{z}}{\partial z}=-i k_{x} E_{x}(z)
$$

Using the time dependence of the $\vec{E}$ field and the corresponding wave equation, $\vec{\nabla}^{2} \vec{E}-\frac{\varepsilon}{c^{2}} \frac{\partial^{2} \vec{E}}{\partial t^{2}}=0$, it can be shown that

$$
\frac{d^{2} E_{x}}{d z^{2}}-\alpha^{2} E_{x}=0
$$

where

$$
\alpha^{2}=k_{x}^{2}-\varepsilon \frac{\omega^{2}}{c^{2}}
$$

Thus, $E_{x}(z)$ can be expressed as

$$
E_{x}(z)=A e^{\alpha z} \mp B e^{-\alpha z}
$$

where $A$ and $B$ are arbitrary constants to be determined by the boundary conditions of Eq. 16. Because of the symmetry of the problem due 


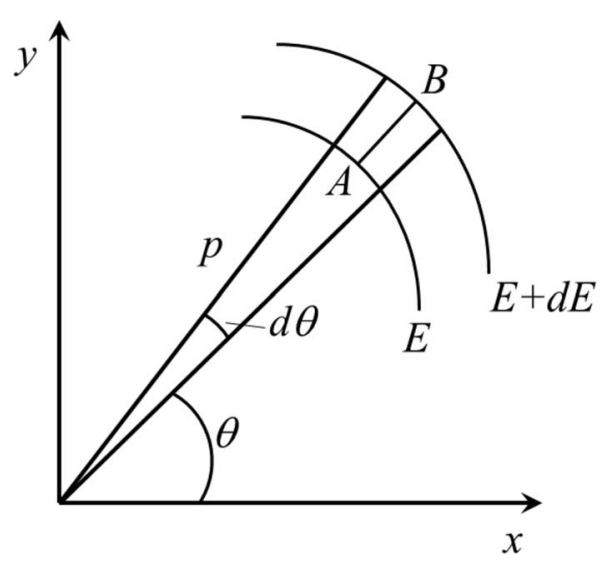

Figure 2. Coordinate system used for the derivations in this work.

to our choice of the coordinate system (Figure 2), we expect $A$ and $B$ to be identical in the medium, 1 . Using the boundary condition in Eq. 12 , one could then show the $\vec{E}$-field in medium 1 to be

$$
\vec{E}_{1}=E_{1}\left\{\hat{i}\left(e^{\alpha_{1} z} \mp e^{\alpha_{1} z}\right)-i \frac{k_{x}}{\alpha_{1}} \hat{k}\left(e^{\alpha_{1} z} \pm e^{-\alpha_{1} z}\right)\right\} e^{i\left(k_{x} x-\omega t\right)}
$$

It is important to note that the $z$-component of the $E$-field is nonzero when $E_{1}$ is non-zero. This component is perpendicular to the propagation vector along the $x$-direction. Thus, the $\vec{E}$-field consists of a purely longitudinal wave and a transverse wave. The $x$-component of the propagation vector $k_{x}$ is the same for both the media. The field along the $x$-direction is truly longitudinal and is associated with the charge oscillation in the metallic layer and can be identified with the surface plasmon waves. The $E$-field is pseudo-transverse because of this longitudinal component.

If $\alpha_{2}$ is real and positive, we expect the $E$-field to be attenuating in medium 2 as expected if the source of this field is the electron cloud oscillating against the positively charged background along the $x$-direction, i.e., the surface plasmon. Then

$$
E_{2 x}=A e^{-\alpha_{2} z}
$$

and

$$
E_{2 z}(z)=i \frac{k_{x}}{\alpha_{2}} A e^{-\alpha_{2} z}
$$

Using the continuity of $E_{x}$ and $D_{z}$ at $z=a$, one obtains

$$
A=\frac{\left(e^{\alpha_{1} a} \mp e^{-\alpha_{1} a}\right)}{e^{-\alpha_{2} a}} E_{1}
$$

and

$$
A=-\frac{\varepsilon_{1}}{\varepsilon_{2}} \frac{\alpha_{2}}{\alpha_{1}} \frac{\left(e^{\alpha_{1} a} \pm e^{-\alpha_{1} a}\right)}{e^{-\alpha_{2} a}} E_{1}
$$

respectively. Eqs. 22 and 23 lead to the dispersion relations of two branches of surface plasmons of a metallic slab,

$$
\frac{\varepsilon_{1}}{\varepsilon_{2}}=\frac{\alpha_{1}}{\alpha_{2}} \frac{\left(e^{-\alpha_{1} a} \mp e^{\alpha_{1} a}\right)}{\left(e^{-\alpha_{1} a} \pm e^{\alpha_{1} a}\right)}
$$

In the limit $a \rightarrow \infty$, one obtains the dispersion relationship of an interface,

$$
k_{x}^{2}=\frac{\omega^{2}}{c^{2}} \frac{\varepsilon_{1} \varepsilon_{2}}{\varepsilon_{1}+\varepsilon_{2}}
$$

Eq. 25 leads to

$$
k_{1 z}=\frac{\omega}{c}\left(\frac{\varepsilon_{1}^{2}}{\varepsilon_{1}+\varepsilon_{2}}\right)^{1 / 2}=-i \alpha_{1}
$$

and

$$
k_{2 z}=\frac{\omega}{c}\left(\frac{\varepsilon_{2}^{2}}{\varepsilon_{1}+\varepsilon_{2}}\right)^{1 / 2}=i \alpha_{2}
$$

If both $\varepsilon_{1}, \varepsilon_{2}>0, k_{1 z}$ and $k_{2 z}$ will be real, and the associated electromagnetic fields will correspond to waves of propagation vector $k_{1,2}=\left(k_{x}^{2}+k_{1,2}^{2}\right)=\left(\frac{\omega}{c}\right)^{2} \varepsilon_{1,2}$. For a metallic slab, this will be true for $\omega>\omega_{p}$ where $\omega_{p}$ corresponds to the plasmon frequency. For $\omega<\omega_{p}, \varepsilon_{1}<0$ and $\varepsilon_{1}+\varepsilon_{2}<0$. In this case, $\alpha_{1}$ and $\alpha_{2}$ could be real and positive. The field will be attenuating along the $z$-direction and the propagation vector will be only along the $x$-direction. This is the spectral regime of interest for the present study on the effect of surface plasmons on the radiating ions in the vicinity of the metallic surface. It is also interesting to examine the short wavelength limit of Eq. 25,

$$
\varepsilon_{1}=-1
$$

when we assume that the second medium is vacuum. Using the Drude model of metals for the frequency dependence of the dielectric constant of a metal, one obtains the frequency, $\omega_{s p}$ at which Eq. 28 is satisfied,

$$
\omega_{s p}=\frac{\omega_{p}}{\sqrt{2}}
$$

This characteristic frequency is referred to as the surface plasmon polariton frequency.

So far, we have been discussing the plasmon dispersion relation in the limit $a \rightarrow \infty$, i.e., the interface of a dielectric medium and metal. For a metallic slab of finite thickness, Eq. 24 implies two branches for the dispersion relations, i.e.,

$$
\varepsilon_{1} k_{2 z}+\varepsilon_{2} k_{1 z} \tanh \left(\frac{k_{1 z} a}{i}\right)=0
$$

and

$$
\varepsilon_{1} k_{2 z}+\varepsilon_{2} k_{1 z} \operatorname{coth}\left(\frac{k_{2 z} a}{i}\right)=0
$$

For thin films with a finite thickness of $2 a$, these equations need to be used for the dispersion behavior of the surface plasmons.

\section{Radiative Lifetime of an Optically Active Ion Near a Metallic Surface}

Our main objective is to determine how the lifetime of an optically active ion is modified in the presence of a metallic surface. The current understanding of fluorescence from an optically active ion near a metal was briefly summarized by Ford and Weber. ${ }^{28}$ At a distance far greater than $\sim 10^{3} \AA$ from the metallic surface, an excited atom returns to the ground state radiating to the vacuum modes. The corresponding lifetime will be described by $\tau_{R}$. As the distance decreases to the order of the emission wavelength or slightly larger, the radiative lifetime oscillates due to interference of the emitted radiation and that reflected from the metallic surface. This phenomenon was first observed by Drexhage $^{26}$ and was theoretically explained by several authors. ${ }^{29-31}$ As the distance decreases further to the order of $\sim 50 \AA$ to $100 \AA$, the radiative ion starts experiencing the electromagnetic field associated with surface plasmons. This decay has been observed experimentally and discussed earlier. ${ }^{32-35}$ Within this distance, an excited ion radiates not only to the vacuum modes but also to the surface plasmon modes. In a linear approximation, one could write the total excited state lifetime, $\tau$ as

$$
\frac{1}{\tau}=\frac{1}{\tau_{R}}+\frac{1}{\tau_{S P}}\left(+\frac{1}{\tau_{N R}}\right)
$$

In Eq. 32, $\tau_{R}$ refers to lifetime due to pure radiative relaxation, $\tau_{S P}$ to lifetime associated with interaction with the plasmonic modes and $\tau_{N R}$ to all other relaxation methods of the ion that end in loss of the excited state energy. The last term is very critical if any decrease in 
lifetime will be accompanied by an increase of quantum efficiency of the optical ion. At very small distances, $\sim 10 \AA$ or less (comparable to van der Walls radii), the interaction between the optical ion and the metallic surface will be too strong to be described by a canonical model of a metallic surface and an isolated atom. The metal surface and the optical ion have to be considered as a strongly coupled system.

In this manuscript, our focus is to develop a theory of $\tau_{S P}$ using a simple semi-classical model. For a two level fluorescence model of an atom in vacuum, the Einstein coefficients, $A$ and $B$ provide a simple recipe for calculating the radiative lifetime, $\tau_{R}$,

$$
\frac{1}{\tau_{R}}=(A+B \bar{E}(\nu))
$$

In Eq. 33, $\bar{E}(\nu)$ denotes energy density per unit frequency. The coeffcients $A$ and $B$ can be expressed in terms of differential mode density, $\bar{M}(v)$ in vacuum,

$$
\frac{A}{B}=\frac{8 \pi h v^{3}}{c^{3}}=\bar{M}(\nu) h \nu
$$

In order to derive Eq. 34, we have used

$$
\bar{M}(\nu)=\frac{8 \pi v^{2}}{c^{3}}
$$

One could also show that

$$
\bar{E}(\nu)=\bar{M}(\nu) n h \nu
$$

In Eq. 36, $n$ represent number of photons per mode. Using Eqs. 33,34 and 36 , it can be shown that ${ }^{36}$

$$
\frac{1}{\tau_{R}}=B \bar{M}(\nu) h v(1+n)
$$

The coefficient, $B$ can be calculated using quantum mechanical methods ${ }^{37}$ and can be shown to be

$$
B=\frac{2 \pi}{3 \hbar^{2}}\left|\mu_{12}\right|^{2}
$$

for electric dipole transitions of optical ions with randomly oriented transition moments, $\mu_{12}$ given by

$$
\vec{\mu}_{12}=e\langle 1|\vec{r}| 2\rangle
$$

where $|i\rangle, i=1,2$ correspond to the two states between which dipole transitions are taking place.

Eq. 37 provides a very general formulation of lifetime of a radiating ion that would be appropriate for calculating $\tau_{S P}$. We first make a conjecture that

$$
\frac{1}{\tau_{S P}}=B \frac{\bar{M}_{S P}(\nu)}{L(\omega, z)} h \nu(1+n)
$$

We will restrict our discussions henceforward to an infinitely thick metal, instead of a slab of thickness, $2 a$. Therefore, we need a length parameter, $L(\omega, z)$ to account for the fact that plasmon modes will be confined to the $x y$-plane chosen to be the surface of the metallic layer. Since the differential mode density for the surface plasmons will be per unit area, this has to be divided by a length parameter, $L(\omega, z)$ in order to use a relationship similar to Eq. 37 to calculate $\tau_{S P}$. Eq. 40 will then be dimensionally correct. Additionally, we have also assumed that this length parameter will have an explicit dependence on the location, $z$ of radiating atom with respect to the metallic surface because of the attenuating field. ${ }^{38}$ We could have separated this explicit $z$-dependence by another function. However, this form is chosen in anticipation of a classical formulation of $\tau_{S P}$ that we would be using to derive the functional form of $L(\omega, z)$.

It is important to note that $n$ in Eq. 40 is necessary for induced transitions. For the current problem, this will be set to zero. We will consider only the lifetime associated with the spontaneous transition, i.e.,

$$
\frac{1}{\tau_{S P}}=B \frac{\bar{M}_{S P}(\nu)}{L(\omega, z)} h \nu
$$

In the following sections, we determine the functional forms of various terms in Eq. 41 using a semi-classical approach.

\section{Calculation of Differential Mode Density for the Surface Plasmons, $\bar{M}_{S P}(v)$}

The plasmon problem is a two dimensional problem. All the plasmon modes will be confined to the metal dielectric interface which will be chosen as the $x y$-plane (Figure 2). In the previous discussions, we assumed that the $x$-direction as the direction of propagation for the plasmon waves. The plasmon wavevector will now lie in the $x y$-plane. For the electromagnetic waves, we will describe the component of the wavevector lying in this plane as $\vec{p}$. We define the wavevector, $\vec{k}_{j}, \vec{p}$ and $\vec{q}_{j}$ in the medium, $j$ as

$$
k_{j}^{2}=\varepsilon_{j}\left(\frac{\omega}{c}\right)^{2}, q_{j}=\varepsilon_{j}\left(\frac{\omega}{c}\right)^{2}-p^{2}, \overrightarrow{\mathrm{k}}_{j}=\vec{p}+\vec{q}_{j}
$$

A radius vector, $\vec{r}$ will be then

$$
\vec{r}=\hat{k} z+\vec{\rho}
$$

The component of the radius vector that lies on the interface is denoted by $\vec{\rho}$. With this choice of the coordinate system, we now proceed to calculate $\bar{M}_{S P}(v)$.

Consider two equi-energy curves in the $x y$-plane with energies $E$ and $E+d E$. In the elemental area $A$ surrounding points $A$ and $B$, let the total number of modes be $d^{2} N$. Then,

$$
d^{2} N=\frac{A}{(2 \pi)^{2}} p d \theta \frac{d E}{\left|\vec{\nabla}_{p} E\right|}
$$

Upon integrating over the polar angle, $\theta$ one obtains

$$
\frac{1}{A} \frac{d N}{d E}=\frac{1}{2 \pi} \frac{p}{\left|\vec{\nabla}_{p} E\right|}
$$

Since $E=h \nu$, one obtains from Eq. 45

$$
\bar{M}_{2}(\nu)=\frac{p}{\left(\frac{d \omega}{d p}\right)}=p \frac{d p}{d \omega}
$$

Eq. 46 is a general expression for the differential mode density in two dimensions when $E$ is not dependent on the polar angle, $\theta$, i.e., the equi-enrgy curves are indeed circles. In order to calculate $\bar{M}_{S P}$ we have to use the appropriate dispersion relation for surface plasmons,

$$
p^{2}=\left(\frac{\omega}{c}\right)^{2} \frac{\varepsilon_{1} \varepsilon_{2}}{\varepsilon_{1}+\varepsilon_{2}}
$$

This equation is same as the Eq. 25 except that in the new coordinate system we have replaced $k_{x}$ by $p$, a wavevector in the $x y$-plane. If we assume that $\varepsilon_{2}=1$, i.e., the dielectric medium is vacuum and the metallic medium is not absorptive and its dielectric constant is described by $\varepsilon_{1}=1-\left(\frac{\omega_{p}}{\omega}\right)^{2}$,

$$
p^{2}=\frac{1}{c^{2}} \frac{\omega^{4}-\omega_{p}^{2} \omega^{2}}{2 \omega^{2}-\omega_{p}^{2}}
$$

Then it can be shown that

$$
\frac{d \omega}{d p}=c \frac{\left(\omega^{2}-\omega_{p}^{2}\right)^{1 / 2}\left(\omega^{2}-\omega_{p}^{2}\right)^{3 / 2}}{2 \omega^{4}-2 \omega_{p}^{2} \omega^{2}+\omega_{p}^{4}}
$$

Using Eqs. 49, and 46, $\bar{M}_{S P}$ can be expressed as

$$
\bar{M}_{S P}(\nu)=\frac{2 \pi \nu}{c^{2}} \frac{2 \nu^{4}-2 v_{p}^{2} \nu^{2}+v_{p}^{4}}{\left(2 \nu^{2}-2 v_{p}^{2}\right)^{2}}
$$

It is interesting to note that $\bar{M}_{S P}$ has a singularity at $\frac{v_{p}}{\sqrt{2}}$ which is readily identified with the surface plasmon frequency. 


\section{Calculation of the Length Parameter, $L(\omega)$ for a Two Dimensional Problem}

Intuitively $L(\omega)$ provides a thickness to modes confined to a two dimensional surface. It defines a modal volume. One could obtain an estimate of $L(\omega)$ if the differential mode density, $\bar{M}$ in 3-dimension is known from

$$
\frac{\bar{M}_{S P}}{L}=\bar{M}
$$

For electromagnetic waves in vacuum, $L(\omega)$ will then be equal to $\frac{\lambda}{2}$. This simple approach will not be applicable in the case of surface plasmons since we have no a priori information on the differential mode density in three dimensions. Therefore, we will estimate $L(\omega)$ from the power transferred by an arbitrarily oriented electric dipole moment to the surface plasmons at a distance, $d$ away using a classical approach originally developed by Ford and Weber. ${ }^{28}$

Classically the Einstein's $A$ coefficient can be obtained from power dissipated by an electric dipole by

$$
A=\frac{1}{\tau}=\frac{P}{\hbar \omega}
$$

Consider an oscillating dipole moment, $\vec{\mu} e^{-i \omega t}$ located at distance, $(0,0, d)$. At the site of the dipole moment, $\vec{E} e^{-i \omega t}$ is the field produced by a current source associated with the dipole

$$
\vec{j}(\vec{r}, t)=-i \omega \vec{\mu} e^{-i \omega t} \delta(\vec{r}-\hat{k} d)
$$

Then the power dissipated by this dipole will be

$$
P=\frac{\omega}{2} \operatorname{Im}\left(\vec{\mu}^{*} \cdot \vec{E}\right)
$$

Now we have to calculate $\vec{E}$ produced by the current density given in Eq. 53. First, we expand the associate electric and magnetic fields as

$$
\vec{E}(\vec{r})=\int d^{3} \vec{k} e^{i \vec{k} \cdot \vec{r}} \vec{E}_{\vec{k}}
$$

and

$$
\vec{B}(\vec{r})=\int d^{3} \vec{k} e^{i \vec{k} \cdot \vec{r}} \vec{B}_{\vec{k}}
$$

Using the Maxwell Eqs. 5-8 and the Fourier expansions in Eqs. $55-56$, one could show

$$
\vec{E}_{\vec{k}}=-\frac{1}{2 \pi \varepsilon_{1}}\left\{\vec{\mu}+\frac{\vec{k} \times(\vec{k} \times \vec{\mu})}{k^{2}-k_{1}^{2}}\right\} e^{-i k_{z} d}
$$

Using Eq. 57 in Eq. 55, one obtains

$$
\begin{aligned}
\vec{E}(\vec{r})= & -\frac{1}{\pi \varepsilon_{2}} \\
& \int d^{2} \vec{p} e^{i \vec{p} \cdot \vec{p}}\left\{\delta(z-d) \mu_{\perp}+\frac{i}{2 q_{2}} \vec{k}_{2} \times\left(\vec{k}_{2} \times \vec{\mu}\right) e^{i q_{2}(z-d)}\right\}
\end{aligned}
$$

In Eq. 57, $\mu_{\perp}$ denotes the $z$-component of the dipole moment. We will use $\mu_{\|}$to denote the component of the dipole moment parallel to the $x y$-plane. Using Eqs. 54 and 58, one can obtain the dissipated power after integrating over all the directions of $\vec{p}$ and over the domain $\left[0, k_{2}\right]$ where only the second term in Eq. 58 leads to imaginary value of $\vec{\mu}^{*} \cdot \vec{E}$. Then, $P$ can be shown to be

$$
P=\frac{\omega}{2 \varepsilon_{2}} \int_{0}^{k_{2}} d p \frac{p}{q_{2}}\left[p^{2} \mu_{\perp}^{2}+\frac{1}{2}\left(k_{2}^{2}+q_{2}^{2}\right) \mu_{\|}^{2}\right]
$$

The integration in Eq. 59 is simple and yields

$$
P=|\mu|^{2} \varepsilon_{2}^{1 / 2} \frac{\omega^{4}}{3 c^{3}}
$$

Using Eqs. 52 and 60, we obtain,

$$
A_{c}=\frac{16 \pi^{4}}{3 h c^{3}} \varepsilon_{2}^{1 / 2}|\mu|^{2} \nu^{3}
$$

The subscript, $c$ has been used to distinguish it from the quantum mechanical formulation of the $A$ coefficient. $A$ for randomly oriented transition moments in vacuum is given by

$$
A=\frac{64 \pi^{4}}{3 h c^{3}}\left|\vec{\mu}_{12}\right|^{2} \nu^{3}
$$

Thus, $A$ and $A_{c}$ differ by a factor of 4 which can be attributed to the classical definition of the dipole moment. Thus while making a transition from the classical expression to the quantum mechanical equivalent, we need to make this correction for the dipole moment and remember the fact that no averaging has been done to account for the random orientation of the dipole moment in the derivation of $A_{c}$.

The main effect of replacing the vacuum in the half-space, $z<0$ by a metal of refractive index, $\varepsilon_{1}$ is to add the reflected components of the electromagnetic waves from the radiating dipole by the metallic surface at $z=0$ to the $\vec{E}$-field from the dipole in Eq. 58. The reflection coefficients, $r_{21}^{p}$ for the $p$ - and $s$-polarized waves are given by the Fresnel formulas,

$$
r_{21}^{p}=\frac{\varepsilon_{1} q_{2}-\varepsilon_{2} q_{1}}{\varepsilon_{1} q_{2}+\varepsilon_{2} q_{1}}
$$

and

$$
r_{21}^{s}=\frac{q_{2}-q_{1}}{q_{2}+q_{1}}
$$

When the contributions from reflection at the metallic surface to $\vec{E}$-field are included through the reflection coefficients, $\vec{E}_{\text {total }}$ is given by

$$
\begin{aligned}
\vec{E}_{\text {total }} & \\
= & \frac{i}{2 \pi \varepsilon_{2}} \int d^{2} \vec{p} e^{i\left(\vec{p} \cdot \vec{p}+q_{2} d\right)} \\
& \left\{\begin{array}{c}
\vec{\mu} \cdot\left(p \widehat{k}+q_{2} \widehat{p}\right)\left[\left(p \widehat{k}+q_{1} \widehat{p}\right) e^{-i q_{2} z}+\left(p \widehat{k}-q_{2} \widehat{p}\right) r_{21}^{p} e^{i q_{2} z}\right] \\
+k_{2}^{2} \vec{\mu} \cdot \widehat{k} \times \widehat{p} \widehat{k} \times \widehat{p}\left[e^{-i q_{2} z}+r_{21}^{s} e^{i q_{2} z}\right]
\end{array}\right\}
\end{aligned}
$$

This leads to total power, $P$, dissipated by the dipole

$$
P=\frac{\omega}{2 \varepsilon_{2}} \operatorname{Re} \int_{0}^{\infty} \frac{d p}{q_{2}}\left\{\begin{array}{l}
\mu_{\perp}^{2} p^{2}\left[1+r_{21}^{p} e^{i 2 q_{2} d}\right] \\
+\frac{1}{2} \mu_{\|}^{2} k_{2}^{2}\left[1+r_{21}^{s} e^{i 2 q_{2} d}\right] \\
+\frac{1}{2} \mu_{\|}^{2} q_{2}^{2}\left[1-r_{21}^{p} e^{i 2 q_{2} d}\right]
\end{array}\right\}
$$

$P$ can now be partitioned to

$$
P=P_{P H}+P_{S P}+P_{L S W}
$$

$P_{P H}, P_{S P}$ and $P_{L S W}$ refer to power dissipated as photons, surface plasmons and lossy surface waves. We will focus on $P_{S P}$ for $p \geq k_{2}$. The main contribution comes from the pole associated with $r_{21}^{p}$ for $p$ near the plasmon wavevector,

$$
p_{S P}=\frac{\omega}{c}\left(\frac{\varepsilon_{1} \varepsilon_{2}}{\varepsilon_{1}+\varepsilon_{2}}\right)^{1 / 2}
$$

In the vicinity of $p_{S P}, r_{p}^{12}$ can be expanded as

$$
r_{21}^{p}=\frac{2 \varepsilon_{1} \varepsilon_{2}}{\varepsilon_{1}+\varepsilon_{2}} \frac{p_{S P}}{p-p_{S P}}
$$

Evaluating the contribution of this pole to the integral in Eq. 66 using the residue theorem for a contour integration in the upper half of the complex plane, one obtains

$$
P_{S P}=\frac{\pi c^{2}}{\omega}\left[\frac{\left(-\varepsilon_{1}\right)^{1 / 2} p_{S P}^{5}}{\varepsilon_{2}^{3 / 2}\left(\varepsilon_{2}-\varepsilon_{1}\right)}\left(\mu_{\perp}^{2}-\frac{\varepsilon_{2}}{2 \varepsilon_{1}} \mu_{\|}^{2}\right) e^{-2 \alpha_{2} d}\right]
$$


where the subscripts, $\perp$ and $\|$ are used to describe components of the dipole moment perpendicular and parallel to the $x y$-plane of the interface. The surface plasmons are characterized by $p \geq k_{2}$, and are therefore evanescent waves which attenuate as one moves away from the surface. $P_{S P}$ is entirely trapped within the metallic layer. Using Eq. 52 we can write the quantum mechanical form of the plasmon mediated life time, $\tau_{S P}$ as

$$
\frac{1}{\tau_{S P}}=\frac{8 \pi^{2} c^{2}}{h \omega^{2}} \frac{\left(-\varepsilon_{1}\right)^{1 / 2}}{\varepsilon_{2}^{3 / 2}} \frac{p_{S P}^{5}}{\varepsilon_{2}-\varepsilon_{1}}\left(\mu_{\perp}^{2}-\frac{\varepsilon_{2}}{2 \varepsilon_{1}} \mu_{\|}^{2}\right) e^{-2 \alpha_{2} d}
$$

On comparing Eq. 38, Eq. 41 and Eqs. 71, we obtain $B$ to be

$$
B=\frac{2 \pi}{\hbar^{2}}\left(\left(\vec{\mu}_{12}\right)_{\perp}^{2}-\frac{\varepsilon_{2}}{2 \varepsilon_{1}}\left(\vec{\mu}_{12}\right)_{\|}^{2}\right)
$$

before averaging for random orientation.

From Eqs. 41 and 46 , we can express $\tau_{S P}$ as

$$
\frac{1}{\tau_{S P}}=\frac{2 \pi}{\hbar^{2}}\left(\left(\vec{\mu}_{12}\right)_{\perp}^{2}-\frac{\varepsilon_{2}}{2 \varepsilon_{1}}\left(\vec{\mu}_{12}\right)_{\|}^{2}\right) p_{S P} \frac{d p_{S P}}{d \omega} \frac{h v}{L(\omega, d)}
$$

Solving Eqs. 71 and 73 for $L(\omega, z)$ one obtains

$$
L(\omega, d)=\frac{\omega^{3}}{2 c^{2}} \frac{\left(\varepsilon_{2}\right)^{\frac{3}{2}}\left(\varepsilon_{2}-\varepsilon_{1}\right)}{\left(-\varepsilon_{1}\right)^{\frac{1}{2}}} \frac{1}{p_{S P}^{4}} \frac{d p_{S P}}{d \omega} e^{2 \alpha_{2} d}
$$

Now using $p_{S P}$ from Eq. 47, we can express, $L(\omega, d)$ as

$$
L(\omega, d)=\frac{c^{2}}{2 \omega} \frac{\left(\varepsilon_{1}+\varepsilon_{2}\right)^{2}\left(\varepsilon_{2}-\varepsilon_{1}\right)}{\left(-\varepsilon_{1}\right)^{\frac{5}{2}}\left(\varepsilon_{2}\right)^{\frac{1}{2}}} \frac{d p_{S P}}{d \omega} e^{2 \alpha_{2} d}
$$

For a weak dependence of the dielectric constants, $\varepsilon_{1}$ and $\varepsilon_{2}$ on the angular frequency, $\omega$, one obtains from Eq. 68

$$
\frac{d p_{S P}}{d \omega}=\frac{1}{c}\left(\frac{\varepsilon_{1} \varepsilon_{2}}{\varepsilon_{1}+\varepsilon_{2}}\right)^{\frac{1}{2}}
$$

Then, $L(\omega, d)$ simplifies to

$$
L(\omega, d)=\frac{c}{2 \omega} \frac{\left(-\varepsilon_{2}-\varepsilon_{1}\right)^{3 / 2}\left(\varepsilon_{2}-\varepsilon_{1}\right)}{\left(-\varepsilon_{1}\right)^{2}} e^{2 \alpha_{2} d}
$$

$L(\omega)$ depends explicitly on the angular frequency, $\omega$ and the distance of the optical ion from the metal surface, $d$. The first fraction is equal to $\lambda / 2$, same as in the case of vacuum. However, $L(\omega)$ depends implicitly on $\omega$ through the dependence of the dielectric constants of both the media. In the limit, $\left|\varepsilon_{1}\right|>>\left|\varepsilon_{2}\right|$ and $\varepsilon_{1}<0, L(\omega)$ will increase with decreasing dielectric constant of the metal. This will effectively reduce the mode density, $\bar{M}$ in Eq. 51 . This will be compensated by the exponential dependence of $L(\omega, d)$ on $\alpha_{2}$ and inverse dependence of $\alpha_{2}$ on $\varepsilon_{1}$. Thus with increasing $\left|\varepsilon_{1}\right|$ in the domain $[-\infty, 0]$, the spontaneous transition rate to the surface plasmonic modes will increase rapidly, decreasing the lifetime of the excited optical ion as given by Eq. 32. This has been observed for fluorescing ions in the vicinity of metals. Another consequence of the distance dependence of $L(\omega)$ on $d$ is that with increasing separation, $\tau_{S P}$ will increase rapidly with increasing separation between the optical ion and the metallic surface. It is expected that the electric field associated with the surface plasmons attenuates with distance in an exponential manner.

Finally we consider the effect of random orientation of the transition moment of the radiating ion on spontaneous transition. This will lead to

$$
\frac{1}{\tau_{S P}}=\frac{4 \pi^{2}}{3 h L(\omega, d)} p_{S P}^{2}\left|\vec{\mu}_{12}\right|^{2}\left(1-\frac{\varepsilon_{2}}{\varepsilon_{1}}\right)
$$

\section{Conclusions}

In this paper, we have addressed how the lifetime of an optical ion will be affected by a metallic film in the vicinity. If the ion could sense the field due to the surface plasmons, i.e., they are located within the attenuation distance, the probability of relaxation to the ground state through the plasmonic modes will increase. Physically, the surface plasmonic mode will enhance the effective mode density to which an ion in the excited state could access; see Eq. 41. The length parameter, by which the 2-dimensional density of surface plasmon modes has to be corrected, has been addressed in a semiclassical manner and its dependence on the dielectric constants has been demonstrated.

We also developed and used a very general formulation of the lifetime, based on Einstein's $B$ coefficient, as in Eq. 37 for radiative transitions in 3-dimensions and Eq. 40 for the surface plasmons. The latter is a two-dimensional problem. This approach is often used in laser physics, not so often in the theory of luminescence. This is a powerful approach since we can calculate the lifetime for interaction between a radiating system and another canonical interacting system if the differential mode density is known. The latter is derivable from the appropriate dispersion relation. This approach is easily applied for developing a rigorous quantum mechanical interaction between ions and other perturbing systems such as plasmons, phonons, etc.

We also attempted to show in our introduction how plasmons affect the lifetime and efficiency of a phosphor layer through the surface plasmons excited in a metallic film, and how the measured lifetime and quantum efficiency are not necessarily those of the ion, but of the total combined ion-metallic surface system-a question rarely addressed explicitly in the literature. We showed how important it is to consider an optical ion emitting in a solid as a three-level system, and considered the effect of the plasmons both on the emission and excitation process. We have also shown that it is not correct to assume that any change in the lifetime of an optical ion will have always a positive effect on the overall quantum efficiency. In order to show that an optical device will benefit from a plasmonic modification of its interfaces, one will have to consider explicitly the configuration of the device and its application and the quantum efficiency of the device in the absence of any such modification.

\section{ORCID}

K. C. Mishra (D) https://orcid.org/0000-0003-4081-2858

A. Piquette (D) https://orcid.org/0000-0002-5362-7187

\section{References}

1. W.-H. Chao, R.-J. Wu, C.-S. Tsai, and T.-B. Wu, J. Appl. Phys., 107, 013101 (2010). 2. S. M. Lee and K. C. Choi, Opt. Express, 18, 12144 (2010).

3. E. Cohen-Hoshen, G. W. Bryant, I. Pinkas, J. Sperling, and I. Bar-Joseph, Nano Lett., 12, 4260 (2012).

4. K. Munechika, Y. Chen, A. F. Tillack, A. P. Kulkarni, I. Jen-La Plante, A. M. Munro, and D. S. Ginger, Nano Lett., 11, 2725 (2011).

5. H. Mertens, J. S. Biteen, H. A. Atwater, and A. Polman, Nano Lett., 6, 2623 (2006).

6. A. Gopinath, S. V. Boriskina, S. Yerci, R. Li, and L. Dal Negro, Appl. Phys, Lett., 96, 071113 (2010).

7. C. Jang, S. M. Lee, and K. C. Choi, Opt. Express, 20, 2143 (2012).

8. H. Mertens and A. Polman, Appl. Phys. Lett., 89, 211107 (2006).

9. L. Xu, Y. Qiang, K. Xiao, Y. Zhang, J. Xie, C. Cui, P. Lin, P. Wang, X. Yu, F. Wu, and D. Yang, Appl. Phys. Lett., 110, 233113 (2017).

10. W. Ye, Q. Huang, X. Jiao, X. Liu, and G. Hu, J. Alloy. Compd., 719, 159 (2017).

11. O. L. Muskens and J. Gómez-Rivas, Mater. Sci. Eng. B, 149, 216 (2008).

12. A. Nikitin, M. Remezani, and J. Gómez-Rivas, ECS J. Solid State Sci. Technol., 5, R3164 (2016).

13. M.-K. Kwon, J.-Y. Kim, B.-H. Kim, I.-K. Park, C.-Y. Cho, C. C. Byeon, and S.-J. Park, Adv. Mater., 20, 1253 (2008).

14. K. Okamoto, I. Niki, A. Shvartser, G. Maltezos, Y. Narukawa, T. Mukai, Y. Kawakami, and A. Scherer, Phys. Stat. Sol. A, 204, 2103 (2007).

15. K. Okamoto, I. Niki, A. Shvartser, Y. Narukawa, T. Mukai, and A. Scherer, Nat. Mater, 3, 601 (2004)

16. J. Vučković, M. Lončar, and A. Scherer, IEEE J. Quantum Electron., 36, 1131 (2000).

17. J. Henson, J. DiMaria, E. Dimakis, T. D. Moustakas, and R. Paiella, Opt. Lett., 37, 79 (2012).

18. J. Henson, J. C. Heckel, E. Dimakis, J. Abell, A. Bhattacharyya, G. Chumanov, T. D. Moustakas, and R. Paiella, Appl. Phys. Lett., 95, 151109 (2009).

19. I. Gontijo, M. Boroditsky, E. Yablonovitch, S. Keller, U. K. Mishra, and S. P. DenBaars, Phys. Rev. B, 60, 11564 (1999).

20. N. E. Hecker, R. A. Höpfel, and N. Sawaki, Physica E, 2, 98 (1998).

21. A. Neogi, C.-W Lee, H. O. Everitt, T. Kuroda, A. Tackeuchi, and E. Yablonovitch, Phys. Rev. B, 66, 153305 (2002).

22. G. Lozano, S. R. K. Rodriguez, M. A. Verschuuren, and J. G. Rivas, Light Sci. Appl., 5, e16080 (2016). 
23. H. Raether, Surface Plasmons on Smooth and Rough Surfaces and on Gratings, Springer-Verlag, Berlin Heidelberg (1988).

24. W. L. Barnes, A. Dereux, and T. W. Ebbesen, Nature, 424, 824 (2003).

25. C. Gong and M. S. Leite, ACS Photonics, 3, 507 (2016).

26. K. H. Drexhage, J. Lumin., 1-2, 693 (1970).

27. O. Madelung, Introduction to Solid State Theory, Springer-Verlag, Berlin Heidelberg (1978).

28. G. W. Ford and W. H. Weber, Phys. Rep., 113, 195 (1984).

29. R. R. Chance, A. Prock, and R. Silbey, J. Chem. Phys., 60, 2744 (1974).

30. H. Kuhn, J. Chem. Phys., 53, 101 (1970).

31. H. Morawitz, Phys. Rev., 187, 1792 (1969).
32. W. H. Weber and C. F. Eagen, Opt. Lett., 4, 236 (1979).

33. A. Adams, J. Moreland, P. K. Hansma, and Z. Schlesinger, Phys. Rev. B, 25, 3457 (1982).

34. R. E. Benner, R. Dornhaus, and R. K. Chang, Opt. Commun., 30, 145 (1979).

35. I. Pockrand, A. Brillante, and D. Möbius, Chem. Phys. Lett., 69, 499 (1980).

36. M. Eichhorn and M. Pollnau, IEEE J. Sel. Top. Quantum Electron., 21, 9000216 (2015).

37. D. P. Craig and T. Thirunamachandran, Molecular Quantum Electrodynamics, Dover Publications, New York (1998).

38. A. Archambault, F. Marquier, J.-J. Greffet, and C. Arnold, Phys. Rev. B, 82, 035411 (2010). 